\title{
Nanobiodiversity and Biomimetic Adhesives Development: From Nature to Production and Application
}

\author{
Angelo Espinoza-Ramirez'1, Huberth Fuentes-Rodriguez' ${ }^{1}$ Edson Hernandez-Herrera1,

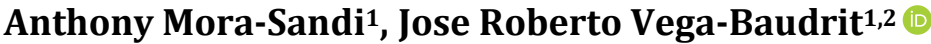 \\ ${ }^{1}$ Chemistry Department, National University of Costa Rica, Heredia, Costa Rica \\ ${ }^{2}$ National Nanotechnology Laboratory LANOTEC-CeNAT-CONARE, San José, Costa Rica \\ Email: jvegab@gmail.com
}

How to cite this paper: Espinoza-Ramirez, A., Fuentes-Rodriguez, H., Hernandez-Herrera, E., Mora-Sandi, A. and Vega-Baudrit, J.R. (2019) Nanobiodiversity and Biomimetic Adhesives Development: From Nature to Production and Application. Journal of Biomaterials and Nanobiotechnology, 10, 78-101. https://doi.org/10.4236/jbnb.2019.102005

Received: November 26, 2018

Accepted: April 1, 2019

Published: April 4, 2019

Copyright $\odot 2019$ by author(s) and Scientific Research Publishing Inc. This work is licensed under the Creative Commons Attribution International License (CC BY 4.0).

http://creativecommons.org/licenses/by/4.0/

\begin{abstract}
Advances in science and technology, especially in bioprospecting and biomimetics, have provided solutions to everyday problems over time. Through bioengineering, research and the discovery of the mechanisms present in nature for the production and functionality of adhesives, different materials and substances capable of imitating the role of their analogs in nature have been developed, which generates positive consequences in the area of the medical, textile, wood industry, among others. In this review, we present some researches and discoveries that have been made, which focus on the way in which adhesives produced by organisms found in nature are made, such as: marine organisms, plants, land organisms, insects, among others. In addition, different types of adhesives that exist and how they can be produced synthetically to be oriented to several industrial applications are mentioned, too.
\end{abstract}

\section{Keywords}

Bioprospecting, Biomimetics, Bioengineering, Adhesives, Industry

\section{Introduction}

When we talk about technological advances, the improvement of the quality of human life has been the main objective. Throughout history, artifacts have been created and optimized in order to facilitate our daily life. In this bibliographical review, we will discuss technological advances in the study, elaboration and improvement of polymeric adhesives from nanostructures coming from nature.

Biomimetics is the engineering field that solves problems based on biological 
models. Therefore, the basic steps in the biomimetic approach are to recognize: what is needed to solve a problem, what is similar in living nature (plant or animal), what are the functional mechanisms (structural, material, mechanical, optical, physiological or pathological) behind the example that is being studied. The final step is to fabricate artificial materials or structures by physical, chemical or biological methods inspired by nature [1]. Biomimetics is closely related to the concept of bionanotechnology, which have been described by Alvarado et al. as the use of biological systems or its derivatives to manipulate the world at nanoscale [2].

Polymers are actually indispensable for all living organisms, for example, cellulose or chitin as a structural polymer, starch and glycogen as an energetic reservoir polymer. Natural rubbers are harvested from the Hevea brasiliensis trees [3]. Natural Rubbers from $H$. brasiliensis are composed primarily of cis-polyisoprene which due to its excellent physical properties is an important source of natural rubber. They are widely used in tires, seals, and shock absorbers because of their high entropy-elasticity [4].

Solid natural rubber consists of approximately $94 \%$ rubber hydrocarbon and $6 \%$ non-rubber components such as lipids, proteins, carbohydrates, etc. These non-rubber components play an important role in stabilizing the latex particles and in contributing to the outstanding properties on natural rubbers [5]. The adhesion of the natural rubbers is intrinsic low due to the very high molar weight of the no-polar rubber chain (Figure 1 and Figure 2). However, a better adhesive property can be achieved by polymerization with a wide variety of compounds such as polyolefin, copolymerization with vinyl pyrrolidone or vinyl acetate, methylmethacrylate and acrylonitrile, or mixture with polymeric component containing functional groups suitable for crosslinking or fillers addition [6].<smiles>[R]C(=O)OCC(COP(=O)([O-])OCC[N+](C)(C)C)OC([R])=O</smiles>

(a)<smiles>[R]C(=O)OCC(COP(=O)([O-])OCC[NH3+])OC([R])=O</smiles>

(b)

Figure 1. The chemical structures of (a) L'-phosphatidyl choline and (b) phosphatidyl ethanolamine, the phospholipids found in $\mathrm{NR}$ latex where $\mathrm{R}$ and $\mathrm{R}$ are long-chain alkyl groups [5]. 
(a)

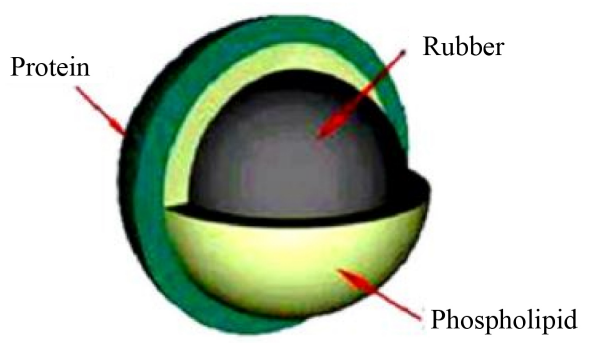

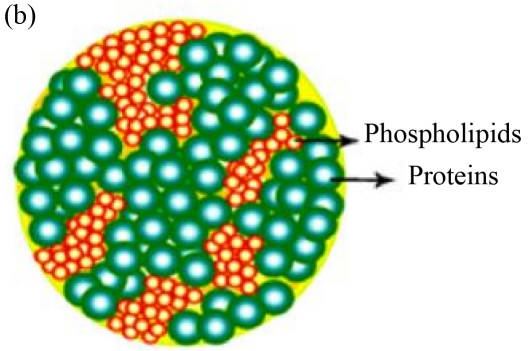

Top view of NR particle

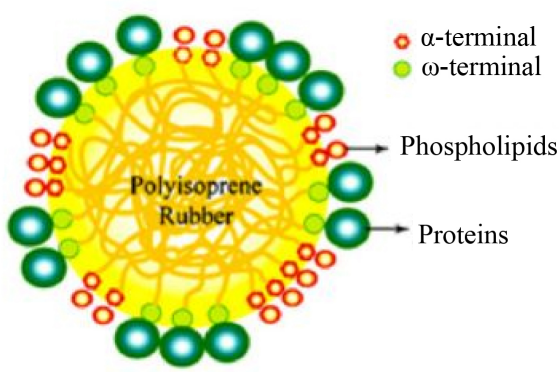

Cross-section of NR particle

Figure 2. Two possible models for the structure of the rubber latex particle surface. (a) A current model of a natural rubber latex particle surrounded by a double-layer of proteins and phospholipids, and (b) the proposed new model consisting of a mixed layer of proteins and phospholipids around the latex particle [5].

Bioadhesives has been simple defined as unmodified natural adhesives used by biological systems for attachment, these has properties that synthetic adhesives do not, like rapid reversible adhesion that allows insects to climb walls, strong weather-resistant adhesion that allows ivy to attach to buildings, and advanced underwater adhesion that allows barnacles to cling to the hulls of ships [7]. Due to this and other properties like low cost, biodegradability, renewability and environmental friendliness, bio-based adhesives have shown remarkable potential in manufacturing industry applications [8].

Other example is the fine hair adhesive system used by insects and lizards to climb wet or dry, vertical and even inverted surfaces. The adhesion is primarily due to short range weak van der Waals interactions between the fine hairs on the adhering surface and the target surface. There are some works that has focused on mimicking the terminal bristle component of the adhesive by fabricating arrays of polymeric nanorods demonstrated expected amounts of adhesion [9]. For example, some synthetic gecko adhesives with dry adhesive function have been fabricated using polymers as well as multiwall carbon nanotubes, however it's hard to achieve a stable adhesion after some contact cycles and none have been shown to function under water [10]. It is reported that multiwall carbon nanotubes improve the average shear strength of the adhesion [11].

Adding several compounds allows to modify or adjust properties of the natural adhesives, but most of the times the new properties comes at expense of others. Introduction of nanotechnology, besides of the allowing of mimic natural adhesive structures, has opened an opportunity for adhesive industry to develop 
a new generation of adhesives [12]. Now we will review several researches about the different types of adhesives present in nature and how with the nanotechnology and by nano-biomimetic it is possible recreate and enhance these different bio-adhesives.

\section{Types of Adhesion}

First of all, we need to understand the two types of adhesion that exist, adhesion and bioadhesion and its respectively mechanisms. Adhesion its molecular attraction between the contacting surfaces of two solid or liquid phases, this can be classified in chemical and physical mechanisms. In short, adhesion refers to the ability of the adhesive to flow, moisten the substrate and maintain certain intermolecular physicochemical forces [13]. The physical adhesion is always present, even if it is weak. This is caused by the van der Waals forces that occur at each interface, often complemented by significant contributions from stronger links that may be present. On the other hand, the chemical mechanism includes possibilities of covalent, ionic, metallic bonding and, in some cases, chelation. There are numerous possibilities to create chemical bonds. [14]

Bioadhesion differs from conventional adhesion from the point of view of the requirements and properties of the substrate(s) being joined; it is governed by interfacial phenomena. Bioadhesion mechanisms are more complex and it cannot be classified in only 2 mechanisms. The types of bioadhesion mechanisms are shown in Table 1 . It is important to mention that the quality of an adhesive is typically determined by evaluating two different physical forces: adhesion, which as we saw earlier is the intermolecular force that maintains the bond between the substrate and the adhesive; and the cohesion, which is the internal resistance of the adhesive or its ability to withstand shear stresses [13].

\section{Nano Biomimetic Adhesives}

The evolution of biological organisms over millions of years has allowed for refined development of bioadhesives, which play a crucial role in the organism's survival. Like typical adhesives, bio-adhesives are desired for their ability to fill cavities and spaces, and join dissimilar materials, using high shear and tensile

Table 1. Bioadhesion mechanisms [13].

Bioadhesion mechanism

Electrostatic: Electrostatic forces between the tissue and the bioadhesive material.

Wetting: Ability of the bioadhesive to spread and develop intimate contact with the target surface.

Adsorption: Surface forces resulting in chemical bonding between the target material and the bioadhesive.

Diffusion: Results in physical entanglements of the tissue and the bioadhesive's flexible polymer chains.

Mechanical: Interlocking of the bioadhesive into irregularities on the rough surface of the tissue. 
strength to generate strong bonding. In recent years, researchers have sought to learn from the principles used in these bio-adhesives to develop new generations of synthetic adhesives built on the fundamental principles exploited by nature. These synthetic adhesives, whose goal is to mimic the function and structure of bio-adhesives, have been termed bio-inspired adhesives or biomimetic adhesives [7]. Of course, from many millions of natural species known to science, only few selected model species have been examined experimentally [15].

\subsection{Inspiration from Plants}

With increasing concerns over environmental threats and sustainable development, deployment of biodegradable and sustainable biomass for the production of wood adhesive and other adhesives that are important in the industry, is not only inevitable but also responsive to reducing the impact caused by formaldehyde adhesives [16]. In addition, as an alternative to avoid the use of formaldehyde adhesives, it is possible to find in the plant kingdom (intrinsic in the different ways of adapting and surviving from some plants and other related organisms) some of the different types of mechanisms for bioadhesion that are found throughout nature, which have been studied over time with the aim of contributing to nanobiomimetics and the development of other types of adhesives with better characteristics and greater efficiency, trying to have a lower cost in comparison with the commonly used today.

Based on the above, researches focused on the characterization of the surfaces of some plants have been developed, since the different existing morphologies due to the variability of cell shapes, and hierarchically superimposed micro and nanostructures of the cell surfaces (mainly wax crystals), and by the formation of multicellular structures [17], are related to the type of mechanical bioadhesion, and in addition, define the boundaries for the well-structured world of solids, and it is surfaces that define their interactions, therefore, they play crucial roles in environmental interactions. This is of particular importance for sessile organisms with large functional surfaces: plants. Green plants cover the terrestrial biomes of our planet and show a stunning diversity of hierarchical surface structures which has been revealed with the help of scanning electron microscopy techniques (SEM) first employed in the 1970s. It is even possible to examine the hierarchical surface structures at the macroscopic scale. On the other hand, the details of structures like wax crystals on their surface are only revealed by scanning electron microscopes [17], whereby, the use and development of electronic microscopy has favored the understanding and implementation (on an industrial scale) of this type of adhesion. For example, a material currently used that represents very well the mechanical adhesion, and which is based on the concept of biomimicry applied from plants, is the hook-and-loop fastener by the Swiss engineer Georges de Mestral in the 1950s, popularly known as Velcro that it is based on burrs (plant) surfaces [17]. Another example where the structure of a surface plays an important role in adhesion is the case of the spatula-shaped 
heads found in some beetles from the family Chrysomelidae. This fibrillar contact structures are located on the tarsi of beetles and based on the study of numerous species of these beetles, it has been previously shown that they are extremely specialized for adhering to smooth surfaces [18].

There is another type of adhesion due to the ability of the bioadhesive to spread and develop intimate contact with the target tissue (wet adhesion), which is mainly based on small volumes of a liquid secretion, which forms thin films in the contact zone and the potential of this liquid to contribution of viscous forces to friction, adhesion, and the contact formation in general [19]. Generally, these types of substances contain specific components in their matrix, such as proteins, which interact electrostatically with the chemical structure of the surface where they are, resulting in an adhesion effect between the organism and a specific object or surface [20]. As an example of this adhesion mechanism, there is a species of terrestrial alga called Prasiola linearis, which uses a simple mechanism of adhesion based on a non-specific protein folding and subsequent aggregation process (Figure 3), now thought to be generic for any polypeptide under appropriate conditions. This non-specific folding forms proteinaceous crossed $\beta$-sheet amyloid fibrils, which are usually associated with neurodegenerative diseases. This mechanism presents a saw tooth mechanical response which is the result of unravelling single or multiple layers of proteins from the intermolecular $\beta$-sheets at the surface of the fibril at some point along its length (it is assumed that the fibril lies approximately parallel to the surface of the adhesive). Furthermore, it is suggested that amyloid protein quaternary structures should be considered as a possible generic mechanism for mechanical strength in a range of natural adhesives and other natural materials due to their many beneficial mechanical features and apparent ease of self-assembly [21].

As another example of this adhesion mechanism, there is the plant known as English ivy (Figure 4), which is known to secrete a yellowish sticky substance

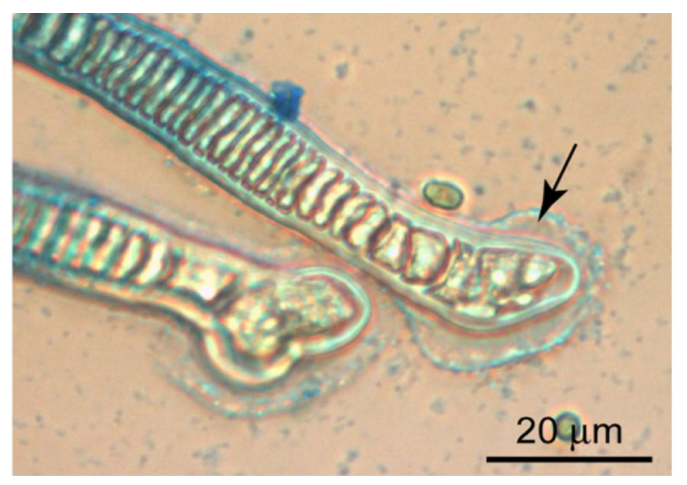

Figure 3. Adhesive observed around the base of $P$. linearis holdfasts. Plants attached to a glass surface were stained briefly with Alcian Blue (1\% in $3 \%$ acetic acid, $\mathrm{pH} 2.5$ ) indicating the presence of polysaccharides. The extent of the extracellular polymeric substances (EPS), the constituent of secreted algal adhesive, can be clearly seen (arrow) [21]. 


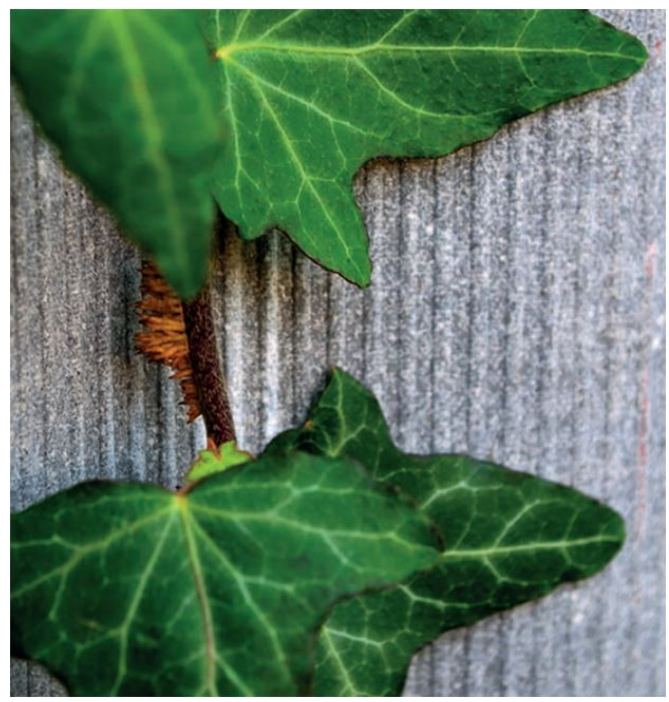

Figure 4. Photograph of the English ivy plant adhered to a vertical surface [22].

when it climbs vertical surfaces. This glue-like secretion, which contains spherical nanoparticles, helps the plant to attach onto surfaces as it climbs. Researchers in the US have now shown that these nanoparticles are composed mainly of arabinogalactan proteins, which are important in promoting strong adhesion. Mingjun Zhang and co-workers-at the Ohio State University, the University of Georgia and the University of Tennessee-isolated the sticky substance from ivy rootlets and characterized it using various microscopy and chemical methods. The nanoparticles are about $70 \mathrm{~nm}$ in diameter and have a negatively charged surface at $\mathrm{pH}$ 7.0. Tests using a phenylglycoside dye confirmed the presence of arabinogalactan proteins-a hydroxyproline-rich glycoprotein typically present in the extracellular matrix of plant cells and other botanic adhesives. The nanoparticles showed low intrinsic viscosity in solution and this gives the adhesive a favorable wetting behavior. Furthermore, due to their size, the nanoparticles are thought to penetrate easily into any surface irregularities, further promoting intimate interactions with the substrate onto which the plant clings. Calcium ions contribute to the curing of the adhesive by promoting electrostatic binding between the nanoparticles and pectin. When the nanoparticles, pectin and calcium ions were combined, the bioadhesive characteristics were reproduced, further validating the adhesion mechanism [22].

On the other hand, we can also find adhesion mechanisms adapted to more difficult environments, such as the seabed. In these habitats are organisms such as brown algae (Figure 5), which produce phenolic compounds that exhibit adhesive properties, and extraordinarily high cohesive strength. These adhesive phenolic compounds bind non-specifically to both hydrophobic and hydrophilic surfaces in aqueous conditions, after their secretion, the polyphenols are activated by a vanadate-peroxidase type of enzyme catalyst that enables the cross-linking of the polyphenol to the extracellular carbohydrate fibers, which 


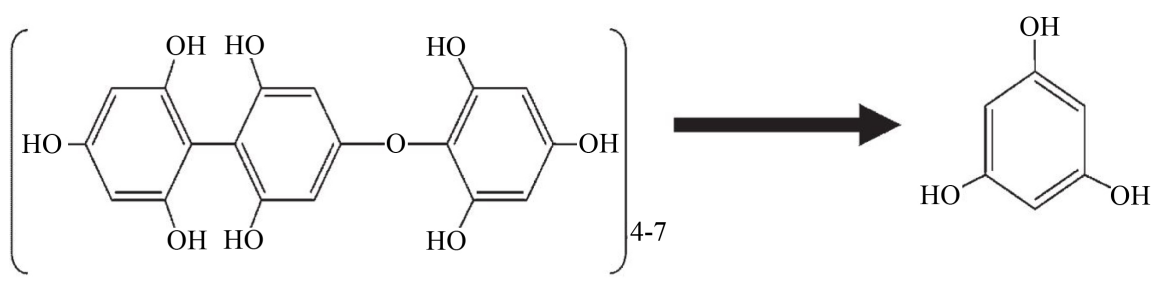

Figure 5. Chemical structure of (a) brown algal polyphenol and (b) phloroglucinol monomer [23].

eventually leads to formation of an algal adhesive. Recently, the nanostructure of adhesive materials extracted from the brown algae Fucus serratus has been investigated by Bitton, $R$., 2008. These adhesive materials are composed of phenolic polymer, alginate, and $\mathrm{CaCl}_{2}$ [23] [24].

In relation to the characterization and manufacture of adhesives inspired by different species of organisms that inhabit nature, there are several methods and techniques that are currently carried out in order to understand and develop these biomimetic adhesives for a common good in the society. Most of these methods are covered by the processes Top-down and Bottom-up, which refer to the reconstruction of a surface or material to elaborate a specific structure (Top-down), as well as the construction of relatively large, complex structural surfaces or materials in molecular to nanoscale by simple, smaller structural units; such as atoms, molecules, nanoparticles and others (Bottom-up). Some of the techniques that are covered by these two methods are: templating, lithography, plasma etching, phase separation, self-assembly, sol-gel method, electrospinning, etc. [25].

As a specific example, numerous processes have been proposed to fabricate different types of dry adhesives with mushroom-shaped tips. In some of these processes, the first step is to generate a mold array that has undercut microholes. For example, it is possible to generate a mold with undercut microholes by photolithography on a UV photoresist to define the microhole array and then perform a DUV (deep UV) exposure on a DUV sensitive material (PMGI) to form the undercut; this involved two chemical developments because two photo-sensitive materials are used. After this process, on the basis of the deep-UV patterning of commercial acrylic with semi-collimated light available from germicidal lamps and careful combination with processing conditions, relatively high-aspect-ratio fibers with overhanging caps can be produced over large areas [26].

\subsection{Inspiration from Sea Organisms}

There has been a huge demand for wet adhesion because hostile wet environments have presented an unsurpassed challenge for dental, medical and industrial applications. For example, since most adhesives, coatings and synthetic sealants suffer deterioration and detachment in the presence of moisture, the latest 
generation of dental medicine, have failed in the past years due to the weak bond between dental resin and dental surfaces, and the Irreversible loss of tooth tissue is unavoidable during the replacement of the defective restoration [27].

Other problem is that the success of synthetic adhesives in a hydrated environment is limited, and typically requires certain treatments to clean the contact surface by the use of chemicals and/or partial dehydration of the contact surface in certain cases. Therefore, synthetic adhesives are rarely used for medical applications. Unlike, many natural adhesives work well under highly hydrated conditions or even under complete immersion in water. A marine environment is like a buffet of invertebrate organisms glued to wet surfaces thanks to specialized adhesives. These adhesives are secreted by marine sessile organisms, such as mussels, barnacles and marine tubular worms, and adhere effectively to almost any hydrated underwater surface [23]. Humans could find many uses for effective underwater adhesives like stopping watery leaks, and in medicine, repairing wet living tissues [28]. That's the reason of the recent researches in bioadhesives produced by sea organisms capable of attach to rocks or shells in the wet environments of the ocean.

\subsubsection{Mussels}

Mussels has specialized mussel adhesive proteins (MAP) that are found at the interface between the bevel wire and the surface. These proteins act as cement, which, after secretion, solidifies rapidly by chemical cross-linking in an insoluble plaque, tying the animal to the surfaces. A predominant feature of MAPs is the presence of L-3,4 dihydroxyphenylalanine (DOPA), a catecholic amino acid formed by the posttranslational modification of tyrosine found in MAPs. DOPA has been implicated in crosslinking reactions that lead to the solidification of the liquid protein tail [29] [30]. Other examples of mussel mimetic polymers polymerized by free radicals have been reported. The polymers derived from the catechinic monomer 3,4-dihydroxystyrene have been synthesized, as have the copolymers of this monomer with styrene or styrene sulfonate. The copolymers provide access to a wide range of physical properties through readily accessible starting materials and preparation methods suitable for mass production [20].

Also, silk fibroin functionalized with catechol has been designed. The potential advantages of silk conjugates compared to existing water-soluble catechol conjugates based mainly on poly (ethylene glycol) (PEG) (Figure 6) backbones are that silk conjugates are processable by water but hydrophobic, which should lead to lower degrees of swelling in water than PEG-based conjugates as well as mechanical reinforcement of the adhesive material through physical cross-linking by formation of $\beta$-sheets between silk chains [31].

\subsubsection{Tube Worms}

A species of worm of the family Sabellariidae, called polychaete worm, is a tube inhabitant and exists in the intertidal zone. These worms secrete a type of adhesive cement to build the tunnels where they live. This adhesive consists mainly of 
(a)

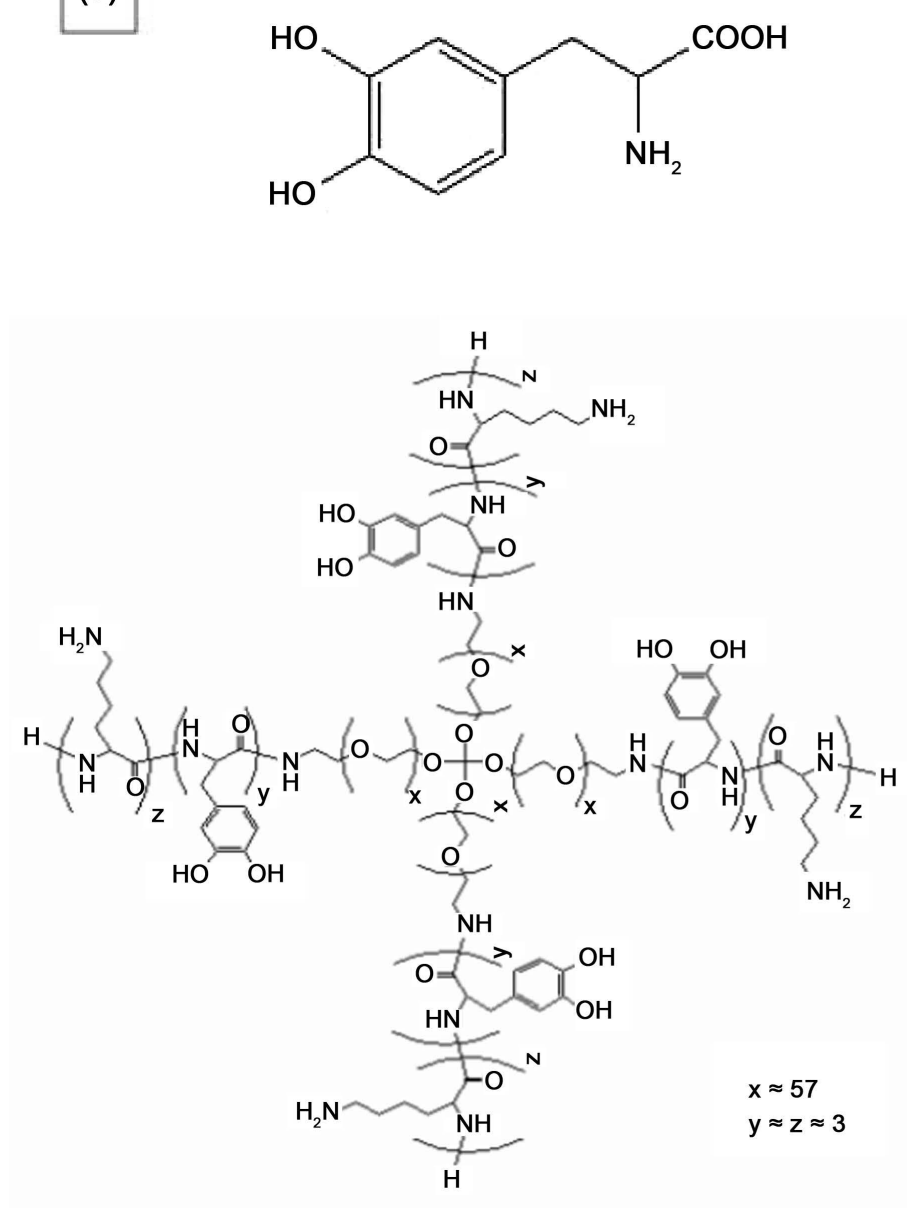

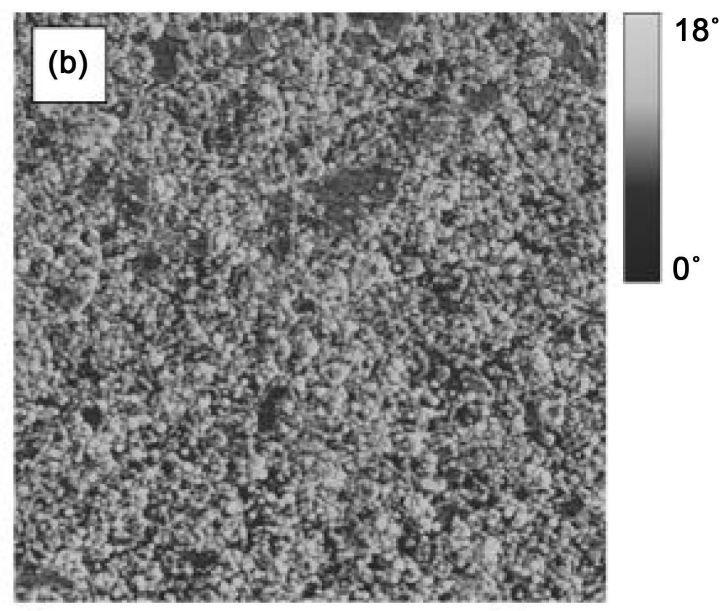
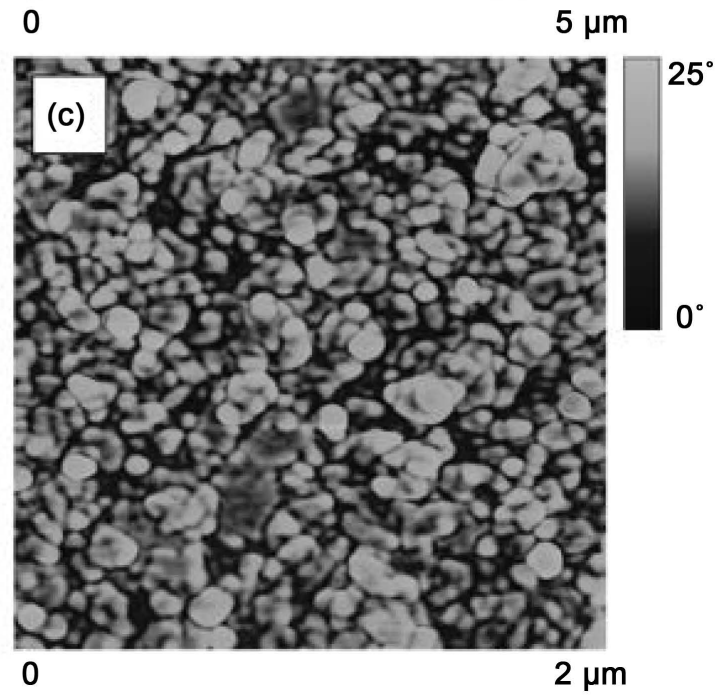

Figure 6. (a) Molecular structure of a single DOPA molecule and DOPA-Lys-PEG polymer. (b), (c) phase AFM images of a single layer of C platelets adsorbed on a layer of DOPA-Lys-PEG \{polymer. The surface roughness (root mean square) is $1.695 \mathrm{~nm}$ in (b) and $1.429 \mathrm{~nm}$ in (c) [29].

three proteins, called cement proteins Phragmatopoma 1-3 (abbreviated as Pc-1, Pc-2, Pc-3) and large amounts of calcium and magnesium ions. These proteins are polyelectrolytes that have opposite charges and are compacted when their $\mathrm{pH}$ is changing. Pc-1 and Pc-2 contain DOPA residues that play the role of adhesion to the surfaces and cross-linking of the adhesive (Figure 7). Studies show that this bioadhesive is degradable, has osteoconductive properties and is useful in the reconstruction of craniofacial fractures [32].

\subsubsection{Barnacles}

In barnacles, adhesion is achieved by the release of a permanent adhesive called cement. This cement consists of $90 \%$ protein, $1 \%$ lipid, $1 \%$ carbohydrate and $4 \%$ inorganic ash. There are more than 10 types of proteins in this cement structure that are called cement proteins and are abbreviated as cps [33]. Three proteins (cp-19k, cp-20k and cp-68k) have surface functions, which means that they connect the cement to the surfaces, two (cp-52k and cp-100k) have bulk functions 


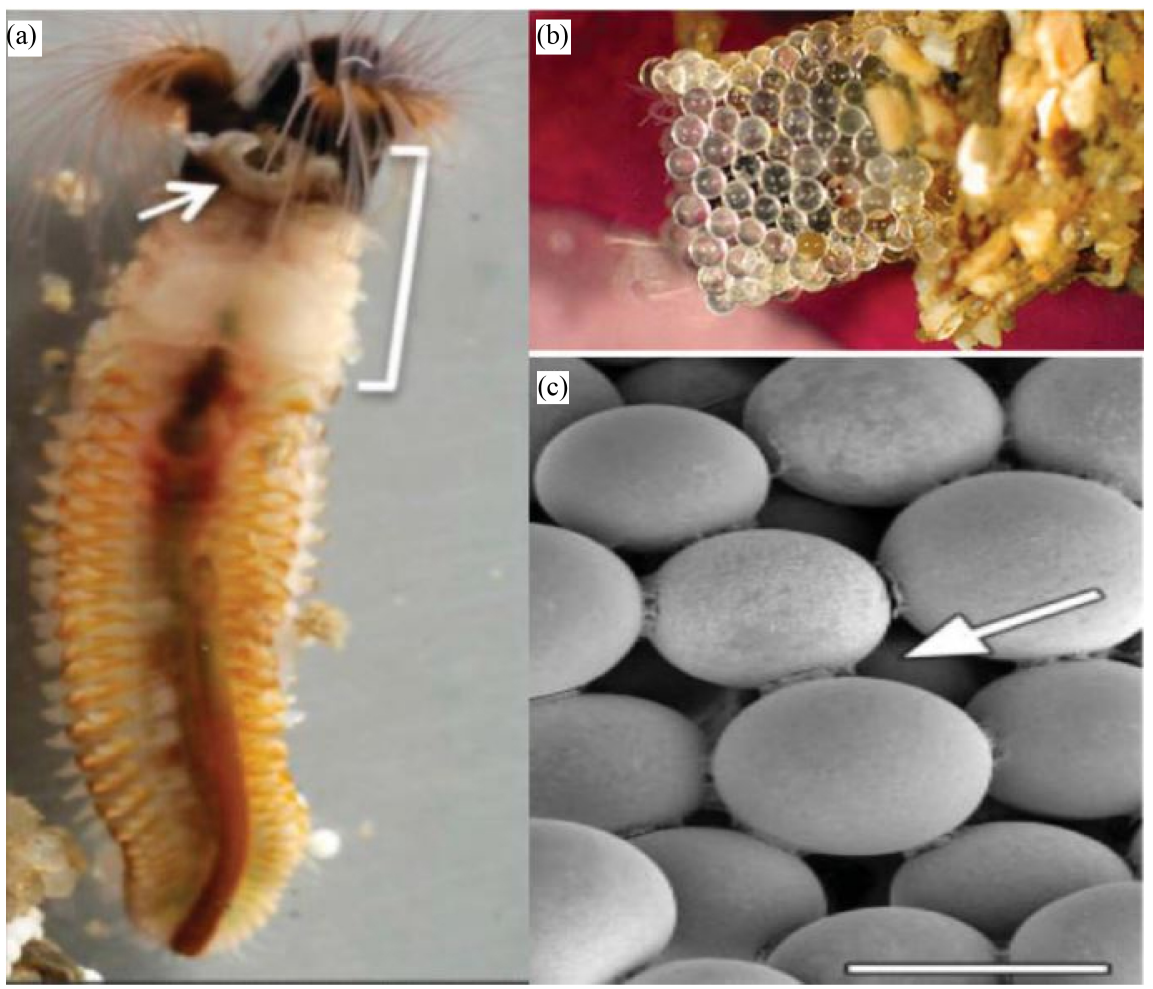

Figure 7. (a) Image of a tube worm. (b) A tube with $0.5 \mathrm{~mm}$ glass beads. (c) Glass beads attached together by cement plaques (shown by arrow) [32].

which means they maintain the cement structure and one (cp-16k) has an enzymatic function that protects the cement from microbial degradation. Unlike mussels and tubular worms, in barnacles the adhesion mechanism does not imply DOPA residues [32]. Figure 8 shows some of the possible applications of the barnacle cement.

\subsubsection{Brown Algae}

Brown algae release a viscous adhesive based on carbohydrates that consists of polysaccharides and glycoproteins. In addition, phenolic polymers play an important role in the adhesion of brown algae to difficult surfaces. The polyphenol composition consists of phloroglucinol units that act similar to the DOPA residues of tubular worms and mussels because they can cross-link by oxidation. In fact, alginate-based adhesives are inspired and are similar to seaweed adhesive. This adhesive has the advantage that it adheres well to hydrophilic surfaces such as collagen sheets and hydrophobic surfaces such as plastic [23]. DOPA can be modified with nanocomposites to improve its properties, among which we canmention a strong water-resistant adhesion to various surface substrates, such as mucin, soft tissues, bones, polymers and various metallic substrates [34].

\subsubsection{Octopus}

It has been reported the design of nanosucker adhesive matrices inspired by octopus suckers, capable of adhering to flat and flat surfaces in dry and humid environments. To construct them, colloidal silica crystals are centrifuged in a 


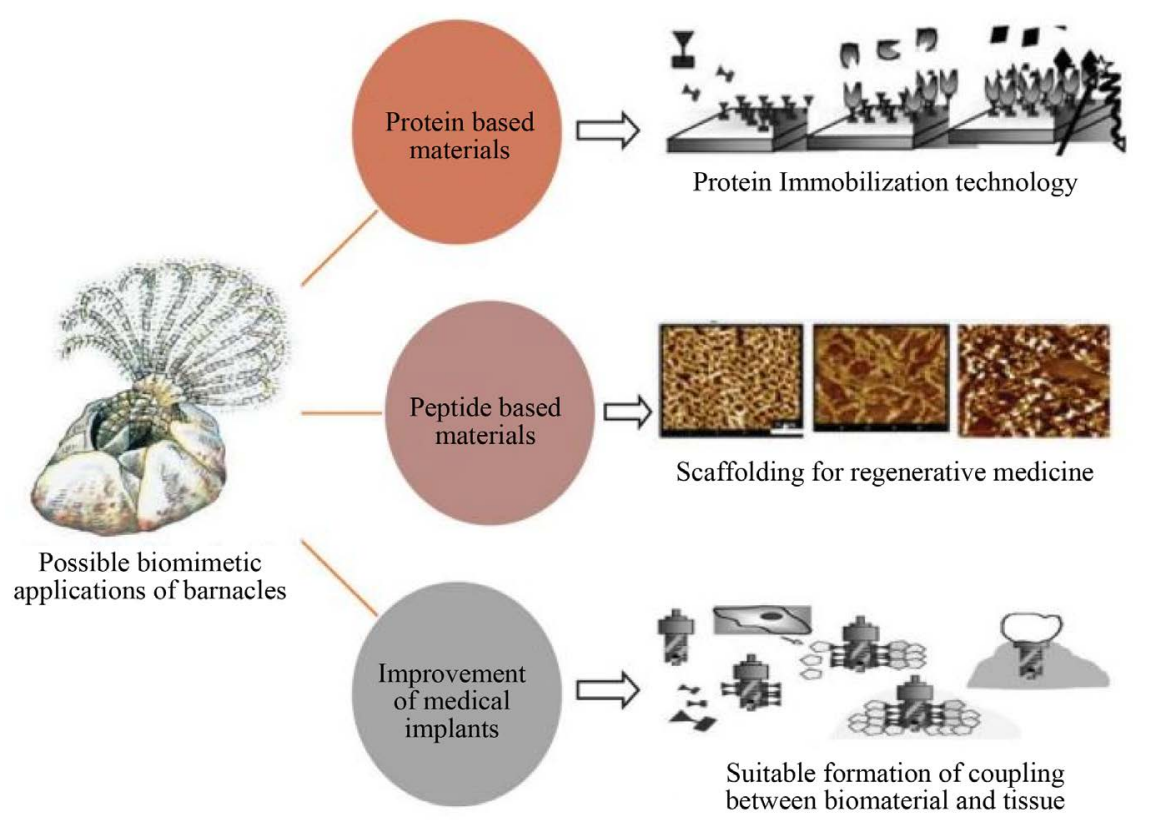

Figure 8. Possible applications of the barnacle cement [32].

non-packaged hexagonal pattern within the ethoxylated trimethylolpropane triacrylate matrix (ETPTA) in the wafer. Subsequently, the silica particles are embedded in a polyvinyl alcohol (PVA) film and released from the ETPTA in the wafer. The molded PVA film then acts as a mold for the manufacture of nanosucker PDMS dies. Also, manufactured flexible nanosuckers can seal on even/irregular surfaces in wet/dry environments driven by van der Waals force and a negative pressure effect when pressed to release internal air. Adhesive matrices exhibit high resistance to both perpendicular and shear forces for multiple cycles. However, the adhesion decreases with time due to the penetration of air (Figure 9) [35].

\subsection{Inspiration from Land Organisms}

\subsubsection{Gecko Inspired Nano Biomimetic Dry Adhesive}

Geckos are exceptional in their ability to scale surfaces of all kinds, due to unusual feature has generated great interest in the last two decades to investigate the structure and mechanism of adhesion of their feet to the surface. Some of the first images of the scanning electron microscope (SEM) from the foot of a Tokay gecko have revealed very complex fiber structures. The gecko-feet have thousands of keratin fibers called setae, which measure between 30 and $130 \mu \mathrm{m}$ long and $5 \mu \mathrm{m}$ in diameter. Each setae branches into hundreds of smaller and thinner fibers (Figure 10). A single foot of the Tokay gecko can produce an adhesive strength of $10 \mathrm{~N}$ per $\mathrm{cm}^{2}$ [36] [37]. These micro and nano fibers ensure good contact with smooth and rough surfaces and are also responsible for the self-cleaning property of gecko-feet. In addition, this branch structure of the setae similar to a tree, is important to adapt a large number of spatulas to rough surfaces and provide a sufficiently large surface in close contact with the substrate 


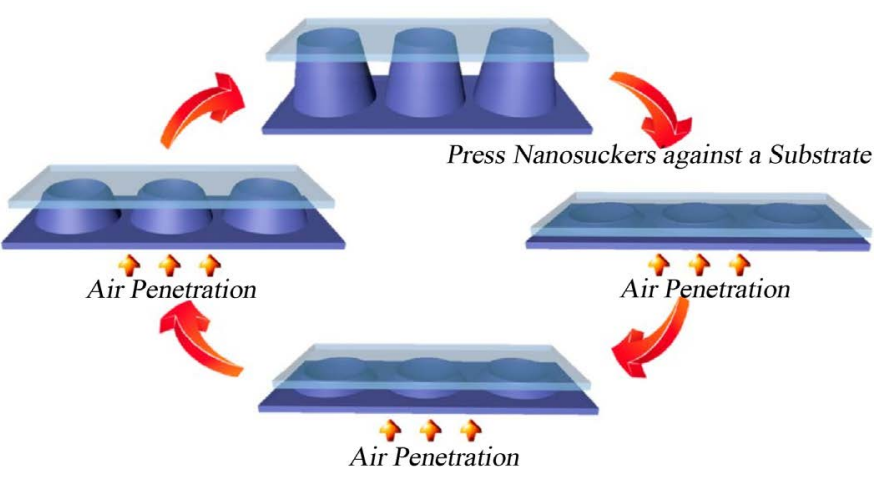

Figure 9. Schematic illustration of the attachment and detachment mechanisms [34].
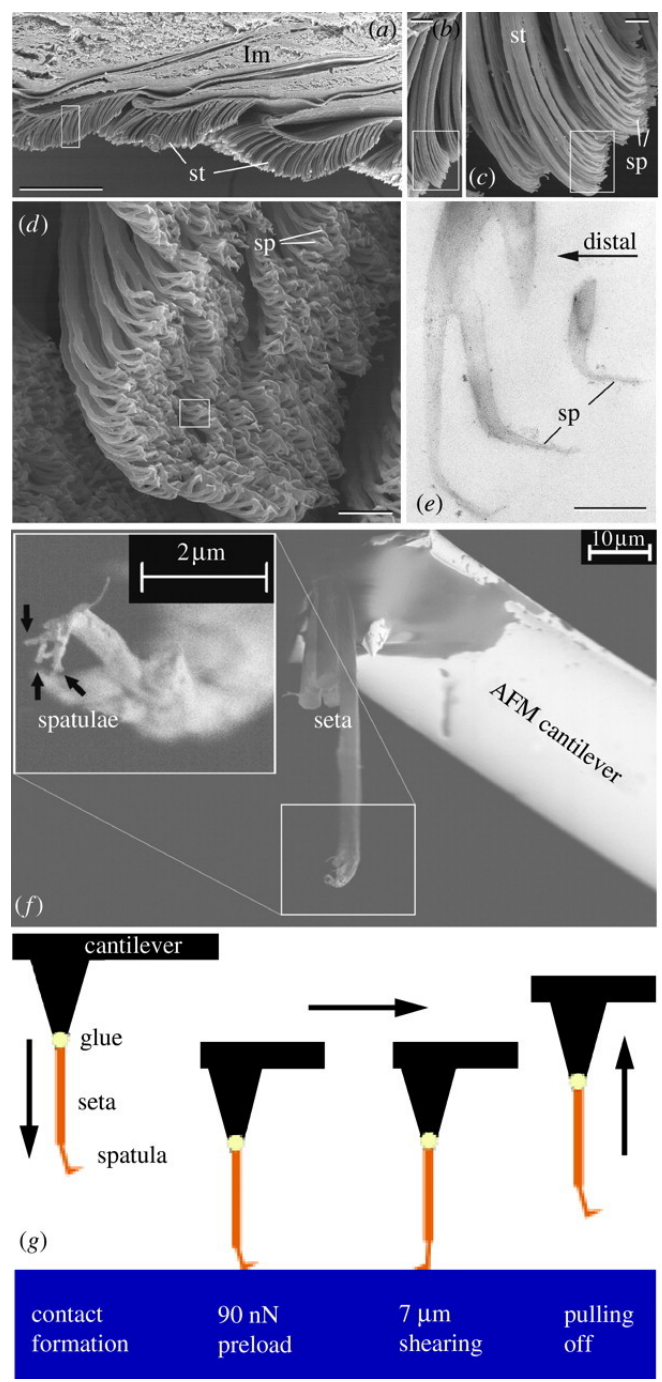

Figure 10. Hierarchical organization of the gecko attachment system (a) longitudinal section of the gecko toe with three lamellae $(\mathrm{lm})$ covered with setae (st) on the ventral side (scale bar, $200 \mathrm{~mm}$ ); (b) setae (scale bar, $10 \mathrm{~mm}$ ); (c), (d) setae branching into spatulae (sp; scale bar, $2 \mathrm{~mm}$ ); (e) spatulae (scale bar, $300 \mathrm{~nm}$; a-d SEM images; e TEM image); (f) single seta with four single spatulae attached to the AFM cantilever (SEM image; $0.5 \mathrm{kV}$ accelerating voltage) [40]. 
and induce sufficient intermolecular forces (van der Waals) for the adhesion [38] [39].

On the other hand, the results have shown that both van der Waals forces and capillary forces play a dominant role in fibrillar adhesion [41]. Some studies have shown that capillary forces influenced the adhesion of Gecko spatulas on hydrophilic substrates in humid environments [38]. Likewise, other investigations discovered that the humidity affected the mechanical properties of the keratin comprising the structure of the spatula, since this made the spatula more flexible, increasing the interaction between the foot of the gecko and the underlying surface. In addition, through these studies, it was shown that van der Waals forces, and not capillary forces, contributed to the strength of the attachment of the gecko's footpad to the surface [7].

These facts inspired scientists to try to mimic the structure of the gecko feet to create strongly dry adhesive materials. Using synthetic polymeric materials, adhesives have been created based on the gecko keratin fiber structures (Figure 11). Thanks to these investigations, design variables have been identified that will determine all the properties mentioned above for synthetic adhesives; these properties are: fiber density, fiber orientation, elastic modulus of fiber and surface energy, and fiber geometry (length, diameter, aspect ratio and tip shape) [36].

Tannouri et al. said that the effectiveness of the dry adhesion is related to these two factors: 1) a high degree of electrostatic interaction between the two surfaces in contact, and 2) the fact that splitting the contact between the surfaces into finner subcontacts increases adhesion. These researchers measure the adhesion force, as shown in Figure 12, lowing a glass sphere to an adhesive surface

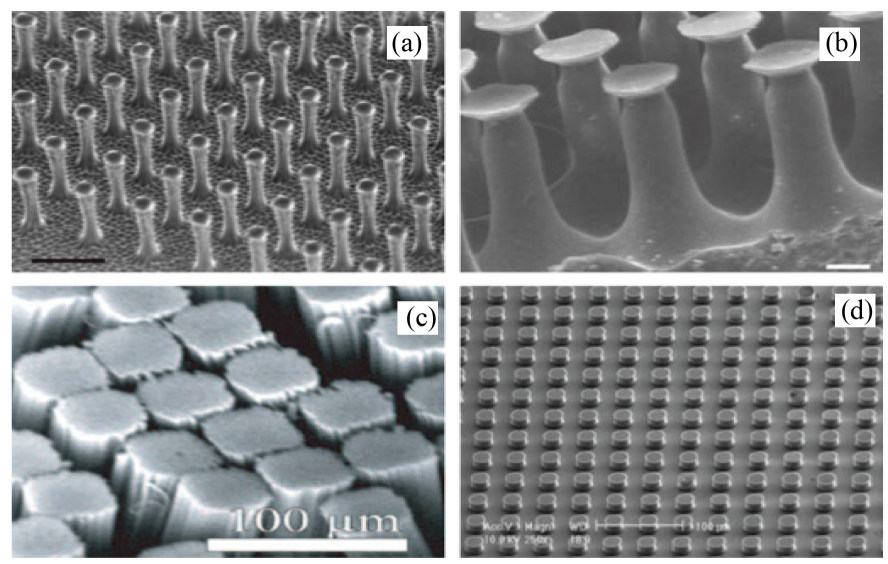

Figure 11. Representative images of bio-inspired adhesives. (a) Scanning electron micrograph (SEM) image of a fabricated self-cleaning, re-attachable dry adhesive inspired by the gecko, scale bar is $2 \mu \mathrm{m}$ [45]. (b) SEM micrographs of fabricated polyvinylsiloxane microscale pillar structures to mimic the biological attachment system of the gecko; pillars are approximately $50 \mu \mathrm{m}$ in diameter and $70 \mu \mathrm{m}$ tall [46]. (c) SEM picture of microfabricated gecko setae tape using carbon nanotube bundles with width of $50 \mu \mathrm{m}$ [47]. (d) SEM image of fabricated stretchable dry adhesive in the form of a wrinkled poly (dimethyl siloxane) patch with micropillars [48]. 

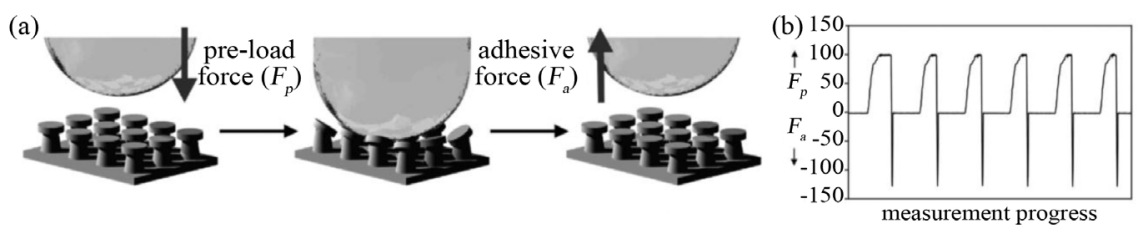

Figure 12. (a) Representation of the measurement of the adhesion between a glass sphere and an adhesive surface with subcontacts. (b) Representative example of macroscale adhesion force data [42].

with a pre-load force and the force required to remove the glass tip from the surface reflects the adhesion between the two surfaces [42].

Surface modification has also been used to create janus abutments for directional adhesion and polymer chains have been grafted onto surfaces to improve material adhesion. Some of the most commonly used synthetic polymer materials for the production of dry adhesives are polydimethisiloxane (PDMS), urethane polyacrylate, polymethyl methacrylate and polyethylene [43].

On the other hand, due to the difficulty of synthetically mimicking the fine structure of the setaes and spatulas of the geckos, these dry polymeric adhesives are not comparable with gecko feet [44]. In addition, nanorod matrices showed reduced adhesion with use, due to clumping and contamination, suggesting the reason for the superhydrophobic nature of the gecko platform [9].

Finally, the ideal properties of a synthetic gecko foot inspired adhesives can be listed as:

- Adhesion through van der Waals interactions.

- Anisotropic adhesion.

- A high pull-off to preload ratio.

- Low detachment force when required.

- Self-cleaning.

- Anti-self-matting/self-adhesion.

- A low to no adhesion state in the absence of shear [49].

\subsubsection{Frog Nano Biomimetic Adhesives}

Tree frogs are organisms less studied than geckos, but no less impressive, since they present even more interesting fixation pads. Unlike geckos, adapted to dry environments, tree frogs can adhere and climb on wet, vertical and salient surfaces without falling. Its pad surface shows a regular hexagonal topography with $10-15 \mu \mathrm{m}$ of epithelial cells separated by channels of $1 \mu \mathrm{m}$ wide. The surface of each epithelial cell is covered by series of densely packed nanopillars $300-400$ $\mathrm{nm}$ in diameter, each with a slightly concave upper surface (Figure 13) [50]. The adhesive mechanism of tree frogs has been extensively investigated, it has been determined that the topography of the surface is important both for the distribution of the fluid through the platform, and for the generation of friction forces. For this reason, arboreal frogs inject a moisturizing liquid into the contact area between the substrate and the pad, which generates an attractive interaction of relatively long range due to the formation of capillary bridges [51]. 

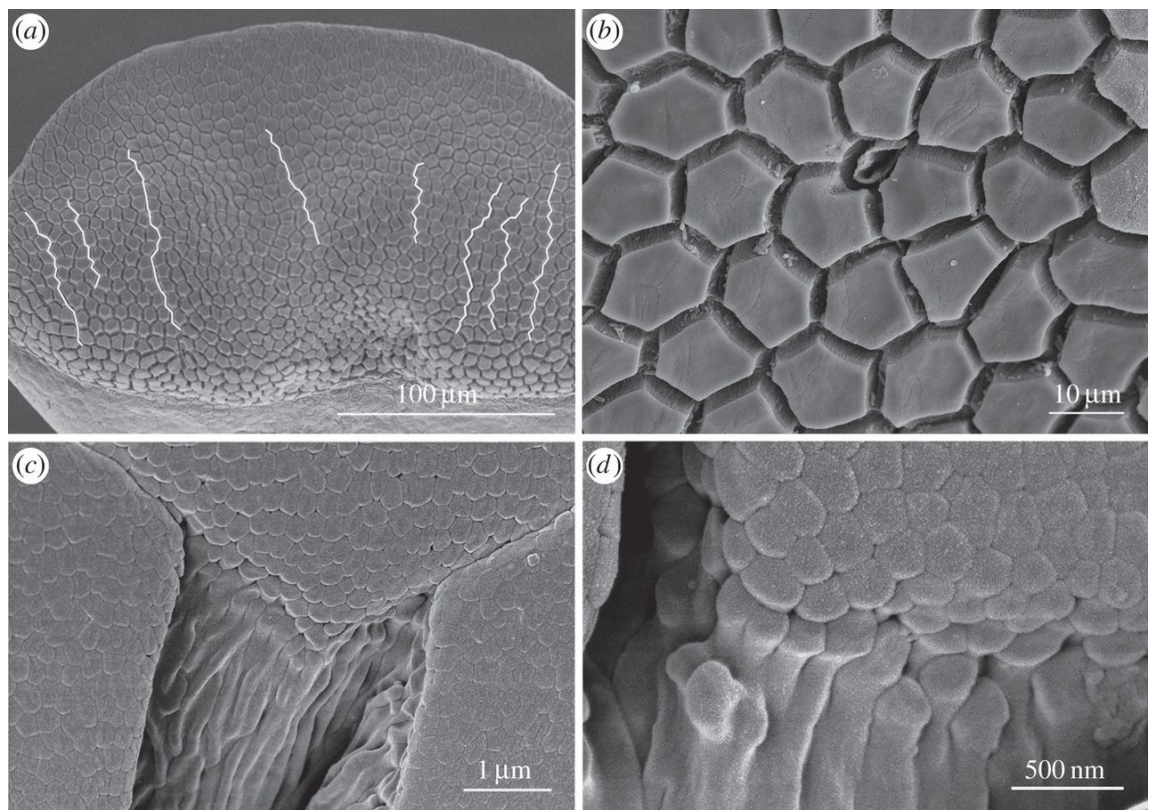

Figure 13. SEM images of toe pad epithelium of $S$. parvus. (a) White lines show relatively straight channels crossing the pad. (b) Polygonal epithelial cells (mainly hexagonal in shape) surrounded by deep channels with a single mucous pore (center). (c) Edge of a pad epithelial cell showing dense array of nanopillars covering the pad surface. (d) High-power view of nanopillars [52].

The beneficial role of surface patterns for attachment under humid conditions has also been demonstrated using imitations of tree frogs by some groups. The patterned surfaces showed higher frictional forces than flat analogs in the presence of a wetting liquid. The principle behind the improved friction was recently determined using frog-type polydimethylsiloxane (PDMS) micropatterns. The results of these tests show that the surface pattern allows drainage of the liquid out of the contact area when a shear force is applied, which is of great importance, because the frog pads work optimally when the layer of fluid under the pad is very thin and there are no air pockets [52].

In addition to allowing excellent drainage, the surface pattern also provides strong direct contact, which indicates that the surface design of the frog's finger pads is specialized for hanging or climbing on wet surfaces, where friction forces come into play [50].

Besides tree frogs, there are also frogs that live around freshwater streams (stream frogs) and waterfalls (rock and torrent frogs) that also have adhesive pads for the toes. Biomechanical studies comparing the adhesive capabilities of rock frogs and tree frogs of comparable size have demonstrated a significantly higher ability of rock frogs to adhere to rough surfaces in the presence of running water. The reported studies on the morphology of their foot pad revealed anatomical differences from the typical pattern of tree frogs. The pads of the epithelial cells of the rock and torrent frogs are more elongated. However, until now there is no experimental evidence that associates elongated patterns with higher adhesion or friction performance in the presence of increasing amounts 
of wetting liquid [50].

On the other hand, frogs have not only been studied by the biomimetic applications of the pads of their legs, as it is the case of the Australian frog (Notaden bennetti) (Figure 14), which secretes a protein-based bioadhesive in the back when it detects a threat. This adhesive is strong, flexible, potentially biocompatible and works very well in wet conditions. Due to all the mentioned characteristics, several interesting evaluations have been made about this adhesive, finding important advantages in different biomedical applications [32]. This adhesive was applied on a sheep cartilage. The results showed that this adhesive has a better effect compared to fibrin/gelatin adhesives and a weaker strength compared to the cyanoacrylate adhesives in this tissue [53]. It is also found that the glue can be used for the repair of the rotator cuff in humans [54].

\subsubsection{Insects Bioadhesives and Biomimetic Arachnid's Adhesives}

Insects and arachnids have developed two clearly different mechanisms to adhere to a variety of substrates, these are, hairy surfaces and flexible and smooth pads [55]. Due to the flexibility of the material of the attachment structures, both mechanisms can maximize the possible contact area with the substrate, regardless of its microsculpture (Figure 15).

These structures are specialized and are not restricted to a particular area of the leg. They can be located in different parts, such as claws, pretarsal derivatives, tarsal apex, tarsomeres or tibia [56]. Some functional principles of mooth pads (adaptability, viscoelasticity, pressure sensitivity) are similar to those known from industrial pressure sensitive adhesion. Hairy attachment pads employed few other features, such as flaw tolerance, lower sensitivity to contamination and roughness, which make them especially interesting from the biomimetic point of view. Hairy attachment systems are typical for evolutionary younger and successful insect groups, such as Coleoptera and Diptera [15].

It has been shown that the density of hairs increases considerably with increasing body weight [55]. Therefore, the increase of the binding force in the

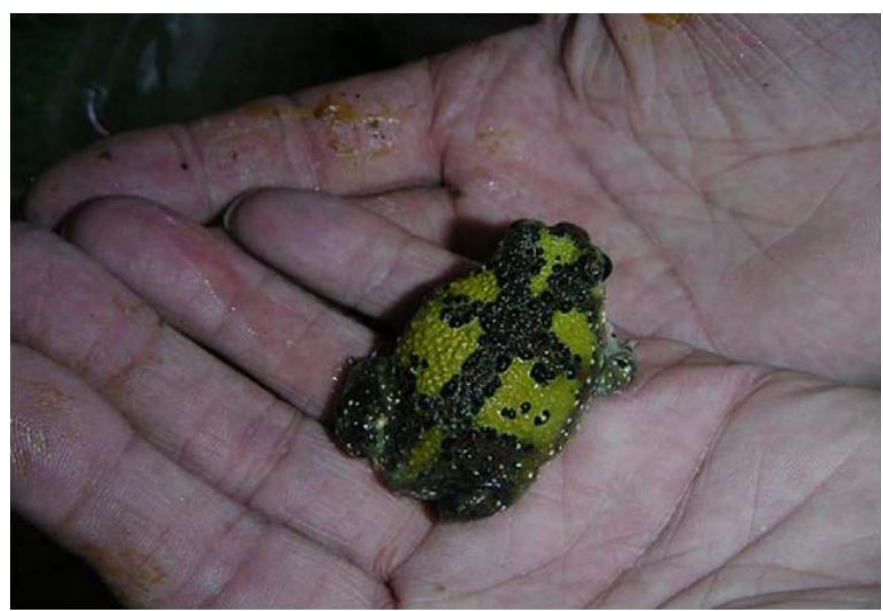

Figure 14. Photograph shows the Notaden benetti frog [54]. 


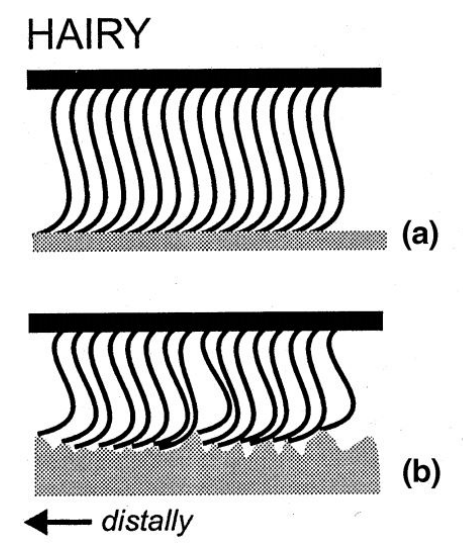

\section{SMOOTH}

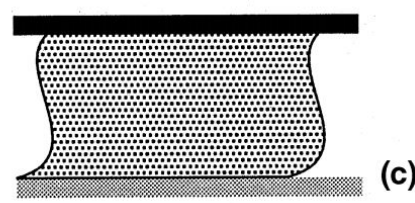

(c)

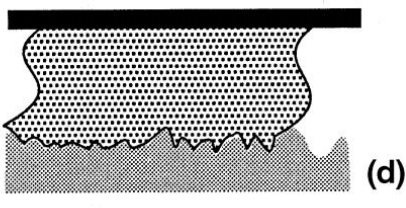

Figure 15. Scheme of action of the "hairy" ((a), (b)) and "smooth" ((c),

(d)) pad attachment systems on the smooth ((a), (c)) and structured ((b),

(d)) substrates. Both systems are able to adapt to the surface profile [55].

hair systems is achieved by increasing the number of individual contact points, that is, by increasing the density of the hair. In addition, animal lineages based on dry adhesion (spiders) have a much higher density of terminal contact elements compared to systems using the mechanism of wet adhesive (insects). Since these effects are based on fundamental physical principles and are mainly related to the geometry of the structure, they should also be applied to artificial surfaces with similar geometry [15].

Despite the differences in morphology, the adhesion in the smooth and hairy pads is mediated by a thin layer of fluid. Adhesive secretions have been found in all the insect groups studied to date, including soft pillows of cockroaches, ants and sticks insects, as well as hairy pads of flies, insects and beetles [57]. This liquid secretion generates an attractive long-range reaction due to the formation of capillary bridges. The liquid injected for insects is an emulsion of the components comprising a lipid fraction and water-soluble nano-drops [55] [58]. This liquid has been optimized for natural selection to moisten most of the surfaces to which the insect must adhere.

The presence of a pad secretion is often used to distinguish between these "wet" adhesives and their "dry" counterparts [19].

On the other hand, until less than a decade ago it was believed that spider hairy fixation systems did not produce fluids, but that van der Waals interactions were responsible for the generation of strong attractive forces; however, it was observed that a layer of water adsorbed on the surface of the solids may further contribute to adhesion in a "dry" adhesive system [15]. A few years later, an investigation revealed clear evidence of pad secretions in different species of spiders (Figure 16) [59]. This leaves the geckos and anoles as the only known representatives of the "dry" adhesive pads in nature [60].

As with the biomimetic studies of geckos and frogs, bioinspired studies in spiders and insects have also developed synthetic adhesives. An example of a biomimetic application was carried out by the Simon Fraser University, they developed a foot design for a spider-inspired scaler robot and examined different 

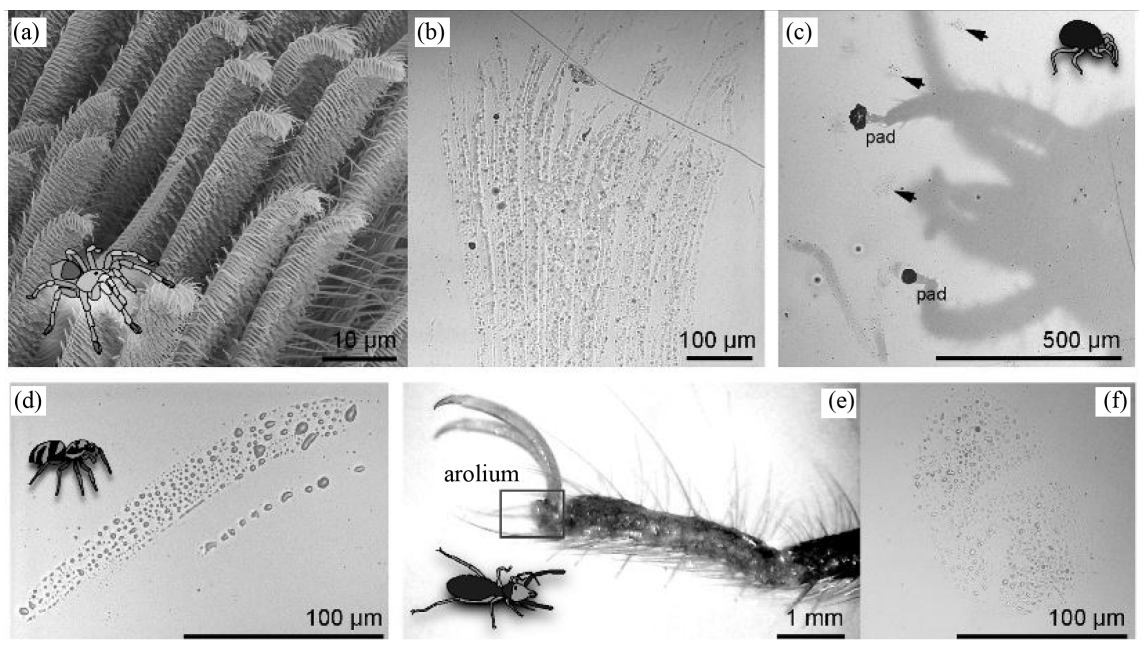

Figure 16. Arachnids investigated for Peattie et al. (2011). (a) Tarantula (Grammostola rosea) setae. (b) Fluid trail left behind by a Grammostola tarsus. (c) Mite (Gromphadorholaelaps schaeferi) clinging upside down to a polystyrene-coated glass coverslip, showing two adhesive pads in contact. Footprints are indicated by arrowheads. A trail of fluid is also visible, lower left. (d) Jumping spider (Salticus scenicus) fluid trail from one tarsus. (e) Solifugid (Gluvia dorsalis) tarsus, arolium situated distally, at base of claws. (f) Fluid footprint left by one Gluvia arolium [59].

factors that affect adherence on differentsurfaces [61]. These climbing robots are used in multiple applications, ranging from automatic cleaning systems for windows and exteriors of buildings, to the inspection of hazardous environments and autonomous vehicles for space applications [61]. Another example of a biomimetic application was made by Gorb et al. (2007), when Patterned Insect inspired by surface polyvinylsiloxane was created (Figure 17).

\section{Conclusion}

The advantages of using nanotechnology to design adhesives inspired by organisms, have become an adequate alternative to old methods of wound closure, such as sutures and staples. The main types of adhesives are based on nature, which include adhesives based on proteins and properties in polysaccharides, synthetic and semi-synthetic adhesives, and biomimetics. The adhesion strength of synthetic and semi-synthetic adhesives is very high, but they have many drawbacks, such as low metabolism and absorption rate, toxic by-products, low adhesion to wet surfaces and prolonged repair time. Taking inspiration from nature leads to wonderful solutions for biomedical adhesive problems. Marine organisms and some animals release adhesives or use different devices found in nano to adhere to surfaces. These adhesives have desirable properties, stories such as high adhesion resistance, reversible adhesion and adhesion to wet and dry surfaces. Given that most of these natural mechanisms are in the process of nanostructures, at present, much attention has been paid to the development of new adhesives with nano/biomaterials inspired by nature. However, it seems impossible to develop a suitable adhesive for all types of surfaces, because 


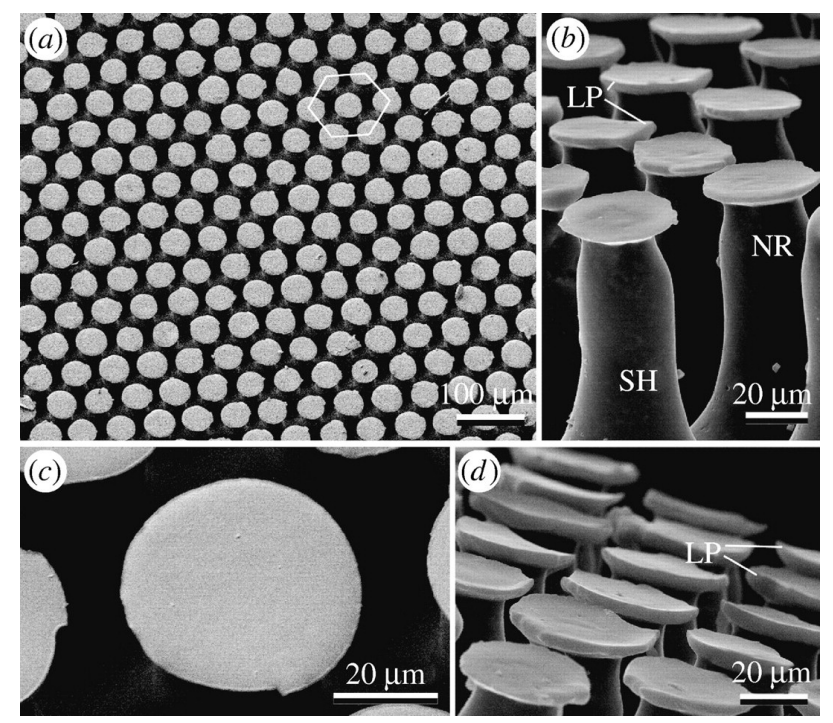

Figure 17. Biomimetic mushroom-shaped fibrillar adhesive microstructure made of PVS. ((a), (c)) View from above; ((b), (d)) Side view. LP, contact plate lip; NR, narrow neck; SH, pillar shaft [18].

they have different functions and properties, even as well as emerging ones, for example, synthetic biology can help consolidate these tools.

\section{Conflicts of Interest}

The authors declare no conflicts of interest regarding the publication of this paper.

\section{References}

[1] Sun, J. and Bhushan, B. (2019) Nanomanufacturing of Bioinspired Surfaces. Tribology International, 129, 67-74. https://doi.org/10.1016/j.triboint.2018.08.007

[2] Alvarado, R. and Baudrit, J.R.V. (2013) Bioinspired Engineering at Nanoscale: Integration of Synthetic Biology and Bionanotechnology. Journal of Bionanoscience, 7, 485-496. https://doi.org/10.1166/jbns.2013.1179

[3] Sun, H., Klok, H.A. and Zhong, Z. (2018) Polymers from Nature and for Nature.

[4] Liu, J., Wang, S., Tang, Z., Huang, J., Guo, B. and Huang, G. (2016) Bioinspired Engineering of Two Different Types of Sacrificial Bonds into Chemically Cross-Linked cis-1, 4-polyisoprene toward a High-Performance Elastomer. Macromolecules, 49, 8593-8604. https://doi.org/10.1021/acs.macromol.6b01576

[5] Nawamawat, K., Sakdapipanich, J.T., Ho, C.C., Ma, Y., Song, J. and Vancso, J.G. (2011) Surface Nanostructure of Hevea brasiliensis Natural Rubber Latex Particles. Colloids and Surfaces A: Physicochemical and Engineering Aspects, 390, 157-166. https://doi.org/10.1016/j.colsurfa.2011.09.021

[6] Rippel, M.M., Costa, C.A.R. and Galembeck, F. (2004) Natural Rubber Latex Modification by Sodium Polyphosphate: A SPM Study on the Improved Latex Adhesion to Glass Sheet. Polymer, 45, 3367-3375. https://doi.org/10.1016/j.polymer.2004.03.013

[7] Favi, P.M., Yi, S., Lenaghan, S.C., Xia, L. and Zhang, M. (2014) Inspiration from the 
Natural World: From Bio-Adhesives to Bio-Inspired Adhesives. Journal of Adhesion Science and Technology, 28, 290-319.

https://doi.org/10.1080/01694243.2012.691809

[8] Wang, Z., Zhao, S., Song, R., Zhang, W., Zhang, S. and Li, J. (2017) The Synergy between Natural Polyphenol-Inspired Catechol Moieties and Plant Protein-Derived Bio-Adhesive Enhances the Wet Bonding Strength. Scientific Reports, 7, 9664. https://doi.org/10.1038/s41598-017-10007-8

[9] Northen, M.T. and Turner, K.L. (2005) A Batch Fabricated Biomimetic Dry Adhesive. Nanotechnology, 16, 1159. https://doi.org/10.1088/0957-4484/16/8/030

[10] Lee, H., Lee, B.P. and Messersmith, P.B. (2007) A Reversible Wet/Dry Adhesive Inspired by Mussels and Geckos. Nature, 448, 338.

https://doi.org/10.1038/nature05968

[11] Hsiao, K.T., Alms, J. and Advani, S.G. (2003) Use of Epoxy/Multiwalled Carbon Nanotubes as Adhesives to Join Graphite Fibre Reinforced Polymer Composites. Nanotechnology, 14, 791. https://doi.org/10.1088/0957-4484/14/7/316

[12] Kaboorani, A. and Riedl, B. (2011) Effects of Adding Nano-Clay on Performance of Polyvinyl Acetate (PVA) as a Wood Adhesive. Composites Part A: Applied Science and Manufacturing, 42, 1031-1039.

https://doi.org/10.1016/j.compositesa.2011.04.007

[13] Khanlari, S. and Dubé, M.A. (2013) Bioadhesives: A Review. Macromolecular Reaction Engineering, 7, 573-587. https://doi.org/10.1002/mren.201300114

[14] Marshall, S.J., Bayne, S.C., Baier, R., Tomsia, A.P. and Marshall, G.W. (2010) A Review of Adhesion Science. Dental Materials, 26, e11-e16.

https://doi.org/10.1016/j.dental.2009.11.157

[15] Gorb, S.N., Sinha, M., Peressadko, A., Daltorio, K.A. and Quinn, R.D. (2007) Insects Did It First: A Micropatterned Adhesive Tape for Robotic Applications. Bioinspiration \& Biomimetics, 2, S117. https://doi.org/10.1088/1748-3182/2/4/S01

[16] Liu, D., Chen, H., Chang, P.R., Wu, Q., Li, K. and Guan, L. (2010) Biomimetic Soy Protein Nanocomposites with Calcium Carbonate Crystalline Arrays for Use as Wood Adhesive. Bioresource Technology, 101, 6235-6241. https://doi.org/10.1016/j.biortech.2010.02.107

[17] Barthlott, W., Mail, M., Bhushan, B. and Koch, K. (2017) Plant Surfaces: Structures and Functions for Biomimetic Innovations. Nano-Micro Letters, 9, 23. https://doi.org/10.1007/s40820-016-0125-1

[18] Gorb, S., Varenberg, M., Peressadko, A. and Tuma, J. (2007) Biomimetic Mushroom-Shaped Fibrillar Adhesive Microstructure. Journal of the Royal Society Interface, 4, 271-275. https://doi.org/10.1098/rsif.2006.0164

[19] Labonte, D. and Federle, W. (2015) Rate-Dependence of "Wet" Biological Adhesives and the Function of the Pad Secretion in Insects. Soft Matter, 11, 8661-8673. https://doi.org/10.1039/C5SM01496D

[20] Lee, B.P., Messersmith, P.B., Israelachvili, J.N. and Waite, J.H. (2011) Mussel-Inspired Adhesives and Coatings. Annual Review of Materials Research, 41, 99-132. https://doi.org/10.1146/annurev-matsci-062910-100429

[21] Mostaert, A.S. and Jarvis, S.P. (2006) Beneficial Characteristics of Mechanically Functional Amyloid Fibrils Evolutionarily Preserved in Natural Adhesives. Nanotechnology, 18, Article ID: 044010. https://doi.org/10.1088/0957-4484/18/4/044010

[22] Chun, A.L. (2016) Bioadhesives: Sticky Plants. Nature Nanotechnology. https://doi.org/10.1038/nnano.2016.126 
[23] Bitton, R. and Bianco-Peled, H. (2008) Novel Biomimetic Adhesives Based on Algae Glue. Macromolecular Bioscience, 8, 393-400. https://doi.org/10.1002/mabi.200700239

[24] Zhong, C., Gurry, T., Cheng, A.A., Downey, J., Deng, Z., Stultz, C.M. and Lu, T.K. (2014) Strong Underwater Adhesives Made by Self-Assembling Multi-Protein Nanofibres. Nature Nanotechnology, 9, 858. https://doi.org/10.1038/nnano.2014.199

[25] Biswas, A., Bayer, I.S., Biris, A.S., Wang, T., Dervishi, E. and Faupel, F. (2012) Advances in Top-Down and Bottom-Up Surface Nanofabrication: Techniques, Applications \& Future Prospects. Advances in Colloid and Interface Science, 170, 2-27. https://doi.org/10.1016/j.cis.2011.11.001

[26] Wang, Y., Hu, H., Shao, J. and Ding, Y. (2014) Fabrication of Well-Defined Mushroom-Shaped Structures for Biomimetic Dry Adhesive by Conventional Photolithography and Molding. ACS Applied Materials \& Interfaces, 6, 2213-2218. https://doi.org/10.1021/am4052393

[27] Ahn, B.K. (2017) Perspectives on Mussel-Inspired Wet Adhesion. Journal of the American Chemical Society, 139, 10166-10171.

https://doi.org/10.1021/jacs.6b13149

[28] Stewart, R.J., Ransom, T.C. and Hlady, V. (2011) Natural Underwater Adhesives. Journal of Polymer Science Part B: Polymer Physics, 49, 757-771. https://doi.org/10.1002/polb.22256

[29] Podsiadlo, P., Liu, Z., Paterson, D., Messersmith, P.B. and Kotov, N.A. (2007) Fusion of Seashell Nacre and Marine Bioadhesive Analogs: High-Strength Nanocomposite by Layer-by-Layer Assembly of Clay and 1-3,4-Dihydroxyphenylalanine Polymer. Advanced Materials, 19, 949-955. https://doi.org/10.1002/adma.200602706

[30] Zhang, H., et al. (2014) Mussel-Inspired Hyperbranched Poly(amino ester) Polymer as Strong Wet Tissue Adhesive. Biomaterials, 35, 711-719. https://doi.org/10.1016/j.biomaterials.2013.10.017

[31] Burke, K.A., Roberts, D.C. and Kaplan, D.L. (2015) Silk Fibroin Aqueous-Based Adhesives Inspired by Mussel Adhesive Proteins. Biomacromolecules, 17, 237-245. https://doi.org/10.1021/acs.biomac.5b01330

[32] Modaresifar, K., Azizian, S. and Hadjizadeh, A. (2016) Nano/Biomimetic Tissue Adhesives Development: From Research to Clinical Application. Polymer Reviews, 56, 329-361. https://doi.org/10.1080/15583724.2015.1114493

[33] Kamino, K. (2008) Underwater Adhesive of Marine Organisms as the Vital Link between Biological Science and Material Science. Marine Biotechnology, 10, 111-121. https://doi.org/10.1007/s10126-007-9076-3

[34] Chen, Y.C. and Yang, H. (2017) Octopus-Inspired Assembly of Nanosucker Arrays for Dry/Wet Adhesion. ACS Nano, 11, 5332-5338. https://doi.org/10.1021/acsnano.7b00809

[35] Sun, W. (2017) Octopus-Inspired Nanosuckers: Adhere to the Rough and Wet.

[36] Shah, G.J. and Sitti, M. (2004) Modeling and Design of Biomimetic Adhesives Inspired by Gecko Foot-Hairs. IEEE International Conference on Robotics and Biomimetics, 22-26 August 2004, 873-878.

[37] Li, Y., Krahn, J. and Menon, C. (2016) Bioinspired Dry Adhesive Materials and Their Application in Robotics: A Review. Journal of Bionic Engineering, 13, 181-199. https://doi.org/10.1016/S1672-6529(16)60293-7

[38] Zhao, A., Mei, T., Lin, X. and Ni, L. (2007) Fabrication and Characterization of Tree-Like Nanorod Arrays for Bionic Gecko Foot-Hairs. 7 th IEEE Conference on 
Nanotechnology, Hong Kong, 2-5 August 2007, 259-262.

[39] Xu, Q., Zhang, W., Dong, C., Sreeprasad, T.S. and Xia, Z. (2016) Biomimetic Self-Cleaning Surfaces: Synthesis, Mechanism and Applications. Journal of the Royal Society Interface, 13, Article ID: 20160300. https://doi.org/10.1098/rsif.2016.0300

[40] Huber, G., Gorb, S.N., Spolenak, R. and Arzt, E. (2005) Resolving the Nanoscale Adhesion of Individual Gecko Spatulae by Atomic Force Microscopy. Biology Letters, 1, 2-4. https://doi.org/10.1098/rsbl.2004.0254

[41] Kim, S. and Sitti, M. (2006) Biologically Inspired Polymer Microfibers with Spatulate Tips as Repeatable Fibrillar Adhesives. Applied Physics Letters, 89, Article ID: 261911. https://doi.org/10.1063/1.2424442

[42] Tannouri, P., Arafeh, K.M., Krahn, J.M., Beaupre, S.L., Menon, C. and Branda, N.R. (2014) A Photoresponsive Biomimetic Dry Adhesive Based on Doped PDMS Microstructures. Chemistry of Materials, 26, 4330-4333. https://doi.org/10.1021/cm502222c

[43] Chaudhary, O.J., Calius, E., Kennedy, J.V. and Travas-Sejdic, J. (2014) Polymer Brushes for Improvement of Dry Adhesion in Biomimetic Dry Adhesives. International Journal of Nanotechnology, 11, 636-644. https://doi.org/10.1504/IJNT.2014.060586

[44] Qu, L. and Dai, L. (2007) Gecko-Foot-Mimetic Aligned Single-Walled Carbon Nanotube Dry Adhesives with Unique Electrical and Thermal Properties. Advanced Materials, 19, 3844-3849. https://doi.org/10.1002/adma.200700023

[45] Geim, A.K., Dubonos, S.V., Grigorieva, I.V., Novoselov, K.S., Zhukov, A.A. and Shapoval, S.Y. (2003) Microfabricated Adhesive Mimicking Gecko Foot-Hair. Nature Materials, 2, 461. https://doi.org/10.1038/nmat917

[46] Bhushan, B. and Sayer, R.A. (2007) Surface Characterization and Friction of a Bio-Inspired Reversible Adhesive Tape. Microsystem Technologies, 13, 71. https://doi.org/10.1007/s00542-006-0256-2

[47] Ge, L., Sethi, S., Ci, L., Ajayan, P.M. and Dhinojwala, A. (2007) Carbon Nanotube-Based Synthetic Gecko Tapes. Proceedings of the National Academy of Sciences, 104, 10792-10795. https://doi.org/10.1073/pnas.0703505104

[48] Jeong, H.E., Kwak, M.K. and Suh, K.Y. (2010) Stretchable, Adhesion-Tunable Dry Adhesive by Surface Wrinkling. Langmuir, 26, 2223-2226. https://doi.org/10.1021/la904290g

[49] Sameoto, D. and Menon, C. (2010) Recent Advances in the Fabrication and Adhesion Testing of Biomimetic Dry Adhesives. Smart Materials and Structures, 19, Article ID: 103001. https://doi.org/10.1088/0964-1726/19/10/103001

[50] Iturri, J., Xue, L., Kappl, M., García-Fernández, L., Barnes, W.J.P., Butt, H.J. and del Campo, A. (2015) Torrent Frog-Inspired Adhesives: Attachment to Flooded Surfaces. Advanced Functional Materials, 25, 1499-1505. https://doi.org/10.1002/adfm.201403751

[51] Persson, B.N.J. (2007) Wet Adhesion with Application to Tree Frog Adhesive Toe Pads and Tires. Journal of Physics. Condensed Matter, 19, Article ID: 376110. https://doi.org/10.1088/0953-8984/19/37/376110

[52] Drotlef, D.M., Appel, E., Peisker, H., Dening, K., del Campo, A., Gorb, S.N. and Barnes, W.J.P. (2015) Morphological Studies of the Toe Pads of the Rock Frog, Staurois parvus (Family: Ranidae) and Their Relevance to the Development of New Biomimetically Inspired Reversible Adhesives. Interface Focus, 5, Article ID: 20140036. https://doi.org/10.1098/rsfs.2014.0036 
[53] Farrar, D.F. (2012) Bone Adhesives for Trauma Surgery: A Review of Challenges and Developments. International Journal of Adhesion and Adhesives, 33, 89-97. https://doi.org/10.1016/j.ijadhadh.2011.11.009

[54] Millar, N.L., Bradley, T.A., Walsh, N.A., Appleyard, R.C., Tyler, M.J. and Murrell, G.A. (2009) Frog Glue Enhances Rotator Cuff Repair in a Laboratory Cadaveric Model. Journal of Shoulder and Elbow Surgery, 18, 639-645. https://doi.org/10.1016/j.jse.2008.12.007

[55] Beutel, R.G. and Gorb, S.N. (2001) Ultrastructure of Attachment Specializations of Hexapods (Arthropoda): Evolutionary Patterns Inferred from a Revised Ordinal Phylogeny. Journal of Zoological Systematics and Evolutionary Research, 39, 177-207. https://doi.org/10.1046/j.1439-0469.2001.00155.x

[56] Speidel, M.W., Kleemeier, M., Hartwig, A., Rischka, K., Ellermann, A., Daniels, R. and Betz, O. (2017) Structural and Tribometric Characterization of Biomimetically Inspired Synthetic “Insect Adhesives". Beilstein Journal of Nanotechnology, 8, 45. https://doi.org/10.3762/bjnano.8.6

[57] Federle, W., Riehle, M., Curtis, A.S. and Full, R.J. (2002) An Integrative Study of Insect Adhesion: Mechanics and Wet Adhesion of Pretarsal Pads in Ants. Integrative and Comparative Biology, 42, 1100-1106. https://doi.org/10.1093/icb/42.6.1100

[58] Li, D., Huson, M.G. and Graham, L.D. (2008) Proteinaceous Adhesive Secretions from Insects, and in Particular the Egg Attachment Glue of Opodiphthera sp. Moths. Archives of Insect Biochemistry and Physiology: Published in Collaboration with the Entomological Society of America, 69, 85-105. https://doi.org/10.1002/arch.20267

[59] Peattie, A.M., Dirks, J.H., Henriques, S. and Federle, W. (2011) Arachnids Secrete a Fluid over Their Adhesive Pads. PLoS ONE, 6, e20485. https://doi.org/10.1371/journal.pone.0020485

[60] Dirks, J.H. and Federle, W. (2011) Fluid-Based Adhesion in Insects-Principles and Challenges. Soft Matter, 7, 11047-11053. https://doi.org/10.1039/c1sm06269g

[61] Sameoto, D., Li, Y. and Menon, C. (2008) Multi-Scale Compliant Foot Designs and Fabrication for Use with a Spider-Inspired Climbing Robot. Journal of Bionic Engineering, 5, 189-196. https://doi.org/10.1016/S1672-6529(08)60024-4 